\title{
Study of FHILs in low luminosity AGNs in the optical spectra
}

\author{
Rafael J. C. Vera ${ }^{1,2}$, Ana M. Rodríguez ${ }^{1,3}$ and J. Gregorio Portilla ${ }^{1,4}$ \\ ${ }^{1}$ Observatorio Astronómico Nacional, Universidad Nacional de Colombia, \\ Carrera 45 No 26-85, Bogotá DC, Colombia \\ ${ }^{2}$ email:rjverar@bt.unal .edu.co \\ ${ }^{3}$ email:anamrodriguezv@unal .edu.co \\ ${ }^{4}$ email:jgportillab@unal .edu.co
}

\begin{abstract}
This work presents the main results from a selection of optical spectra of Seyfert and LINER galaxies taken from the $9^{\text {th }}$ release of the SDSS with detectable coronal emission. A catalogue of 345 Seyfert 1 (Sy1) and Seyfert 2 (Sy2) galaxies with Forbidden High Ionization Lines (FHILs) emission is presented. By analysing their spectra as well as utilising data from the literature we found evidence of anisotropy in optical FHIL emissions between Sy1 and Sy2 galaxies for the following lines: [Nev] $\lambda 3426$, [Fe VII] $\lambda 6087,[\mathrm{Fex}] \lambda 6374$ and [Fe XI] $\lambda 7892$. Results continue indicating that optical FHILs are not observed in LINER type galaxies.
\end{abstract}

Keywords. galaxies: active galaxies: Seyfert galaxies: Liner quasars: emission lines quasars: coronal line

\section{Introduction}

The study of forbidden high ionization lines (FHILs) is important in the study of Active Galactic Nuclei (AGNs) because they can provide important information about the energetic phenomena that is taking place in the innermost region of the active nucleus. In addition, FHILs could give an opportunity to explore relationships with radio, X-rays and other spectral observations. FHILs are better known as coronal lines because they were first detected in the sun's corona; usually are classified as those emissions that arise from chemical species with ionization potential equal or above of $0.1 \mathrm{keV}$. Although other astrophysical objects such as planetary nebula and supernova remnants exhibit emission of FHILs, those coming from AGNs tend to be broader and blue shifted. The presence of FHILs is not common in the spectra of AGNs (an apparition probability of $1 / 3$ ) and, when present, they are relatively difficult to measure due to their low $\mathrm{S} / \mathrm{N}$ ratio. An unusual spectrum of a Sy2 galaxy is shown in Figure 1: in this object all emission lines in the optical are present with relatively high intensity. Although both types of Seyfert galaxies emit FHILs, some authors (Schmitt, 1998; Muruyama \& Taniguchi, 1998; Nagao et al., 2000) have drawn the attention to the possibility of the existence of an anisotropy of coronal emission consisting in a slight excess in Sy1 with respect to Sy2. However, Gelbord et al. (2009) did not find any similar anisotropy in a uniform sample of 63 galaxies with SDSS spectra. This work tries to contribute to this discussion based on a number statistically significant of Seyfert galaxies with coronal emission. Incidentally, due to the searching process of spectra in the SDSS database, we looked for possible LINER galaxies with FHILs emission. 


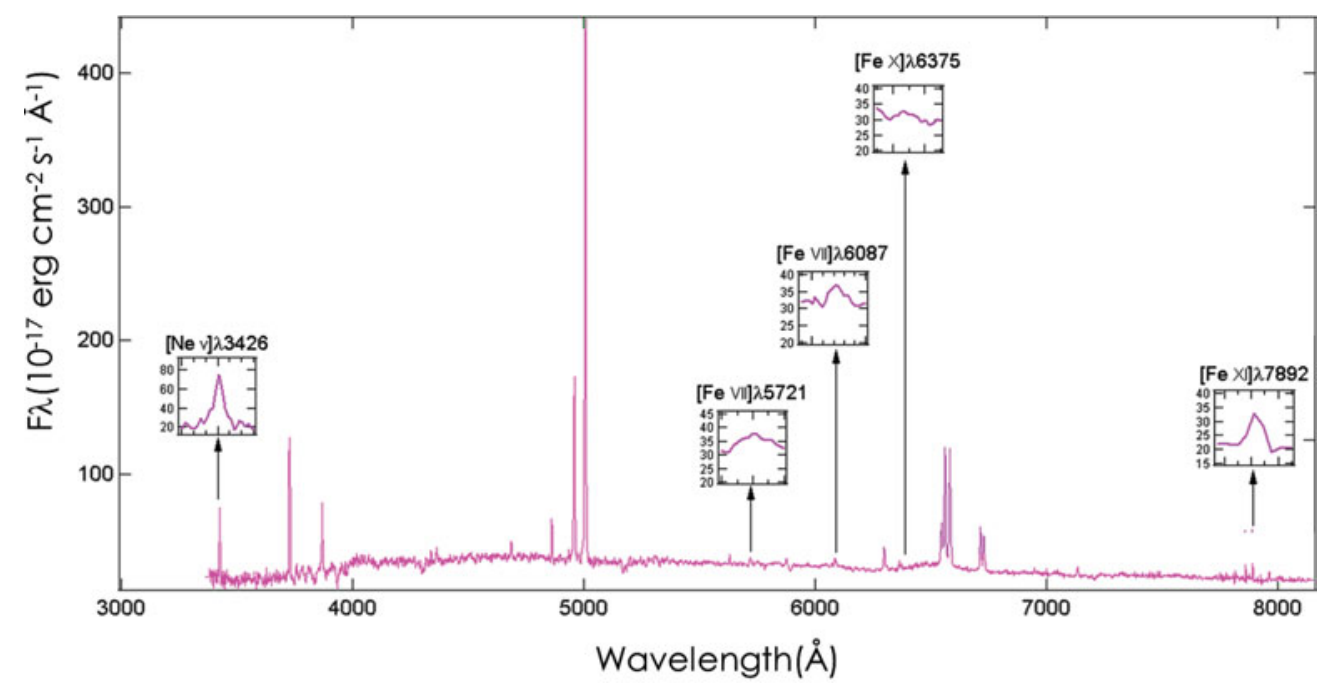

Figure 1. Optical spectrum of the galaxy 2MASX J09452133+1737533. Each line is zoomed for the purpose of clarification. Vera. R (2013) SDSS $9^{\text {th }}$ Rel.

\section{Data Sample}

A fraction of our sample is based on an extensive revision of the literature. In particular the contributions of Muruyama \& Taniguchi (1998), Nagao et al. (2000), Gelbord et al. (2009) and Portilla (2014) were instrumental in assembling the sample. However, because we were interested in spectra with at least two FHILs, as well as in a significant increase in the number of objects for our sample, we proceed to search in the SDSS database through the use of Structured Query Language (SQL) and tailored-search. At the end we gathered information of almost 400 Seyfert galaxies with FHILs emission where more than $85 \%$ of them come from the SDSS database and, consequently, the sample can be considered fairly homogeneous (Fig: 2a). The reader can find detailed information about the search of these data in Vera \& Portilla (2014).

\subsection{LINERs with spectra in SDSS}

Some authors (Dudik et al., 2007; Pereira-Santaella et al., 2010) have reported the presence of coronal lines in LINERs, in particular in the mid-infrared part of the spectrum. Having found strong coronal lines in the optical spectra of Mrk1211b, classified as a LINER by some authors, we examined the possibility to find others LINERs with coronal emission in the optical. Starting from table 3 of the catalog of Véron-Cetty \& Véron (2010) as well as using SQL, we gathered 275 optical spectra of LINER in the SDSS data base.

\section{Results}

In order to examine the possible differences in the FHILs emission between the two types of Seyfert galaxies we proceed to compare the values of fluxes ratios (see Figure $3)$. For each ratio ([Ne V] $\lambda 3426 /[\mathrm{Fe} \mathrm{VII}] \lambda 6087,[\mathrm{FeX}] \lambda 6374 /[\mathrm{Fe} \mathrm{XI}] \lambda 7892,[\mathrm{Fe} \mathrm{VII}]$ $\lambda 6087 /[\mathrm{Fe} \mathrm{X}] \lambda 6374)$ we found anisotropy of emission between the two types of galaxies. Kolmogorov-Smirnov (KS) tests indicate that the null hypothesis of equal distributions is rejected. A distribution of luminosities of FHILs suggests that the observed anisotropy is not due to an inherent deficiency of emission of FHILs for one type of a galaxy with 


\section{Seyfert galaxies}

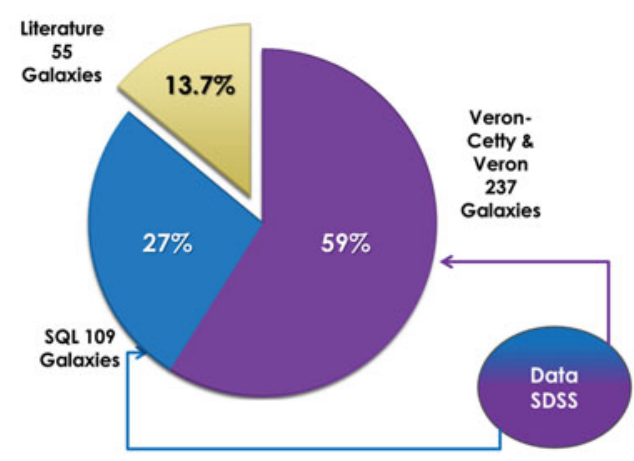

Liners

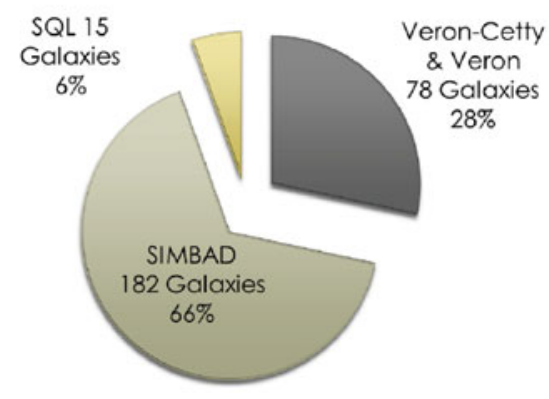

Figure 2. Percentage distribution of the sample. a) Left: Seyfert galaxies ; b) Right: LINERs.

respect to the other (Figure: 4). The only exception is for the [Fe X] $\lambda 6374$ line but this could be explained by the difficulty to isolate this line from the broad component of $\mathrm{H} \alpha$ for Sy1 galaxies.

At the end, we found the following:

- The flux ratios between FHILs suggest anisotropy of emission between Sy1 and Sy2 galaxies, which agrees with the results found by Nagao et al. (2000) and Portilla (2014). Sy1 galaxies seem to emit more FHILs than Sy2 galaxies.
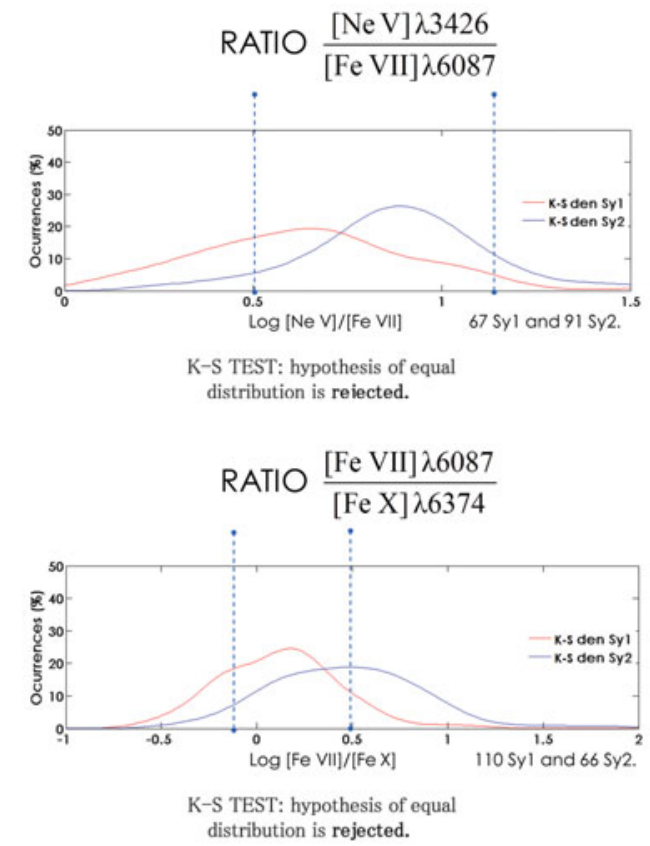

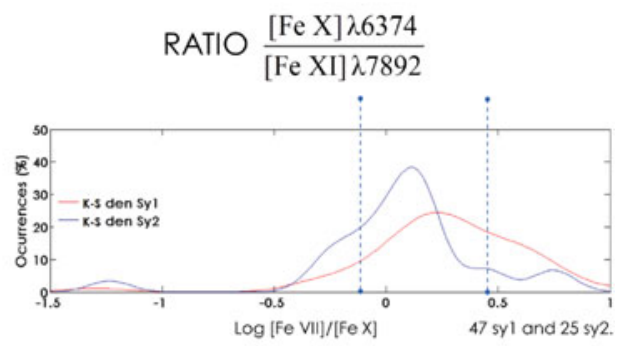

K-S TEST: hypothesis of equal distribution is rejected.

Figure 3. Distribution of the ratios of NeV/Fe VII and Fe VII/ Fe X suggest anisotropy of emission between Sy1 and Sy2 galaxies. 


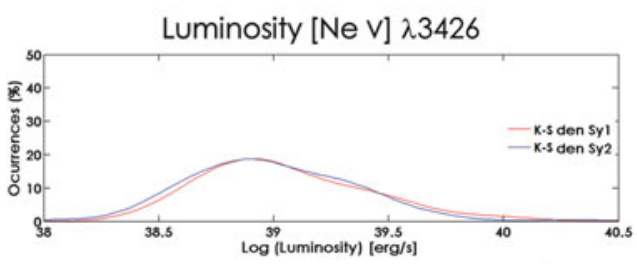

K-S TEST: hypothesis of equal distribution is accepted.

Luminosity [Fe X] $\lambda 6374$

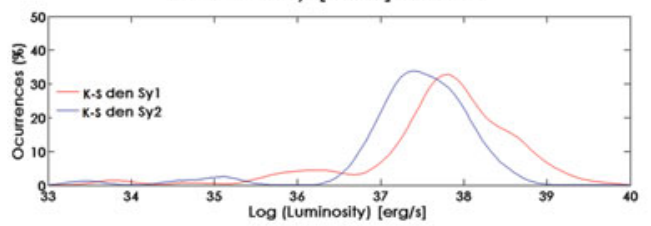

$\mathrm{K}-\mathrm{S}$ TEST: hypothesis of equal distribution is rejected.
Luminosity [Fe VII] $\lambda 6089$

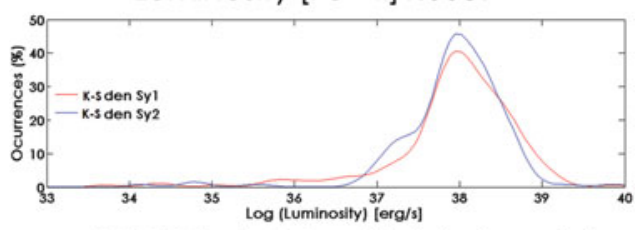

$\mathrm{K}-\mathrm{S}$ TEST: hypothesis of equal distribution is accepted.

Luminosity [Fe XI] $\lambda 7892$

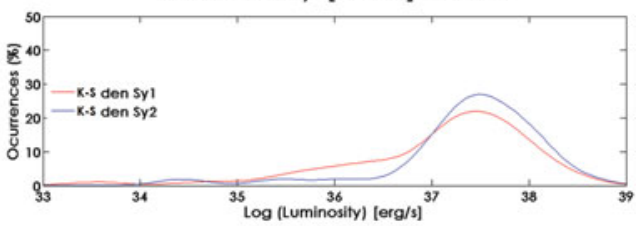

K-S TEST: hypothesis of equal distribution is accepted.

Figure 4. Distribution diagrams for FHILs luminosities.

- Taking in mind the Unified Model, this anisotropy suggests the idea that an important part, but not the majority, of the emission of FHILs comes from the inner part of the obscuring torus.

- It is observed that the ratio of $\mathrm{NeV} / \mathrm{Fe} \mathrm{VII}$ is of the order of 3 to 10 , while the ratios between iron lines (i.e., Fe VII, Fe X, Fe XI) are roughly around the unity.

- We did not find any emission of FHILs in our sample of LINERs. This continues to support the general idea that LINERs are not energetic enough to present optical FHILs.

A complete version of this study including the catalogue with the objects of study, and diagnosis diagrams using only this kind of lines can be found in Vera \& Portilla (2014).

\section{References}

Dudik, R. P., Weingartner, J. C., Satyapal, S., Fischer, J., Dudley, C. C., \& O’Halloran, B. O. 2007, ApJ 664, 71

Ferguson, J. W., Korista, K. T., \& Ferland, G. J. 1997, ApJS, 110, 287

Gelbord, J. M., Mullaney, J. R., \& Ward, M. J. 2009, MNRAS, 397, 172

Korista, K. T. \& Ferland, G. J. 1989, ApJ, 343, 678

Murayama, T. \& Taniguchi, Y. 1998, ApJ 497, L9

Nagao, T., Taniguchi, Y., \& Murayama T. 2000, AJ, 119, 2605

Pereira-Santaella, M., Diamond-Stanic, A. M., Alonso-Herrero, A., \& Rieke, G. H. 2010, ApJ, 725,2270

Portilla, J. G. 2014, (In preparation)

Schmitt, H. R. 1998, ApJ, 555, 663

Vera, R. J. C. 2014, (In preparation)

Véron-Cetty M. \& Véron P. 2010, A\&A, 518, A10. 